\title{
EPR Study of the Phase Transition in Dimethylammonium Gallium Sulfate Hexahydrate
}

\author{
R. Hrabański ${ }^{a}$, M. Janiec-Mateja ${ }^{a}$ And Z. Czapla ${ }^{b}$ \\ ${ }^{a}$ Institute of Physics, Częstochowa University of Technology \\ al. Armii Krajowej 19, 42-200 Czȩstochowa, Poland \\ ${ }^{b}$ Institute of Experimental Physics, University of Wrocław \\ pl. Borna 8, 50-204 Wrocław, Poland
}

\begin{abstract}
$\mathrm{X}$-band electron paramagnetic resonance investigations of single crystals of $\mathrm{Cr}^{3+}$-doped dimethylammonium gallium sulphate hexahydrate are presented from room temperature down to $110 \mathrm{~K}$. The crystal undergoes a order-disorder phase transition to ferroelectric phase at $134 \mathrm{~K}$ and additionally a first-order transition into a low temperature non-ferroelectric phase at $T_{\mathrm{c} 2}=116 \mathrm{~K}$. The spin-Hamiltonian parameters were determined for paraelectric and ferroelectric phases. The spin-Hamiltonian parameters in the paraelectric phase are: $g=1.982 \pm 0.002, b_{2}^{0}=(890 \pm 10) \times 10^{-4} \mathrm{~cm}^{-1}, b=$ $(386 \pm 15) \times 10^{-4} \mathrm{~cm}^{-1}$. Remarkable EPR line width changes confirm the order-disorder character of the ferroelectric phase transition. Additionally observed triplet lines demonstrate freezing-out of dimethylammonium reorientations which can be considered as a prime reason for this transition similarly as observed in dimethylammonium aluminium sulfate hexahydrate.
\end{abstract}

PACS numbers: $64.70 . \mathrm{Kb}, 76.30 . \mathrm{Fc}, 77.80 .-\mathrm{e}$

\section{Introduction}

The crystal structure of dimethylammonium gallium sulfate hexahydrate (DMAGaS) $\left(\mathrm{CH}_{3}\right)_{2} \mathrm{NH}_{2} \mathrm{Ga}\left(\mathrm{SO}_{4}\right)_{2} \cdot 6 \mathrm{H}_{2} \mathrm{O}$ and the isomorphic dimethylammonium aluminium sulfate hexahydrate (DMAAS) is built up of $\mathrm{Ga}$ or $\mathrm{Al}$ cations coordinating six water molecules, regular $\mathrm{SO}_{4}$ tetrahedra and $\left[\left(\mathrm{CH}_{3}\right)_{2} \mathrm{NH}_{2}\right]^{+}(\mathrm{DMA})$ cations, all hydrogen bonded to a three-dimensional framework [1-4]. Both crystals are ferroelastic in the paraelectric phase and showing the monoclinic space 
group $P 2_{1} / n$. Both of them exhibit an order-disorder type transition into a ferroelectric phase at $T_{\mathrm{c} 1}=134 \mathrm{~K}$ and $T_{\mathrm{c} 1}=152 \mathrm{~K}$, respectively. Additionally, only DMAGaS shows a further first-order transition into a low temperature non-ferroelectric phase at $T_{\mathrm{c} 2}=116 \mathrm{~K}$. The reason for this different behavior of DMAAS and DMAGaS is still not fully understood. The ferroelectric transition is associated with the polar DMA cations, which execute hindered rotations in the ferroelastic phase and order in the ferroelectric phase.

Crystals of DMAAS and DMAGaS were widely studied with different experimental methods [1-15]. In particular, the magnetic resonance methods have already been employed in studies of the phase transitions in both systems [9-15]. In the present paper we report on the EPR measurements of chromium doped DMAGaS performed in the X-band $(\approx 9.5 \mathrm{GHz})$ in the temperature range from room temperature $(\mathrm{RT})$ down to $110 \mathrm{~K}$. The rotation pattern of the spectra both in paraelectric and ferroelectric phases are studied in detail and compared. The influence of the dynamics of the polar DMA units on the line shape of EPR spectra is shortly discussed.

\section{Experimental}

Chromium-doped DMAGaS single crystals were grown from aqueous solution containing $1 \mathrm{~mol} \% \mathrm{Cr}_{2}\left(\mathrm{SO}_{4}\right)_{3} \cdot 18 \mathrm{H}_{2} \mathrm{O}$. The trivalent chromium ions with the electron configuration $3 d^{3}$ substitute statistically at gallium sites. The paramagnetic chromium ions are very sensitive probes on the slight changes of their environment.

EPR spectra were recorded in the X-band $(9.5 \mathrm{GHz})$ with a standard spectrometer, equipped with a sample cavity of $\mathrm{TE}_{102}$ mode and $100 \mathrm{kHz}$ modulations. The spectrometer was interfaced with a microcomputer via GPIB board for controlling the spectrometer, acquisition, and display of the acquired spectra.

A goniometer sample holder provided the rotation of the sample $\left( \pm 0.5^{\circ}\right)$ about the given vertical axis. The rectangular laboratory $x y z$ axes frame chosen for the angle-dependent measurements was related to the $\boldsymbol{a}, \boldsymbol{b}, \boldsymbol{c}$ crystallographic axes [3] as follows: $x\|\boldsymbol{a}, y\| \boldsymbol{b}, z \| \boldsymbol{a} \times \boldsymbol{b}$. The spectra were recorded by varying the orientation of the external magnetic field in given plane at $5^{\circ}$ angle intervals. The temperature of the sample was established by regulation of the temperature of cool nitrogen streaming the sample and was controlled by the use of WILDMAD cryostat with an accuracy of relative and absolute temperature values of 0.1 and $1 \mathrm{~K}$, respectively.

\section{Results and discussion}

Angular dependence of the EPR spectra were studied at RT (paraelectric phase) and at $125 \mathrm{~K}$ (ferroelectric one) in three planes perpendicular to the $x$-, $y$-, 
( a )
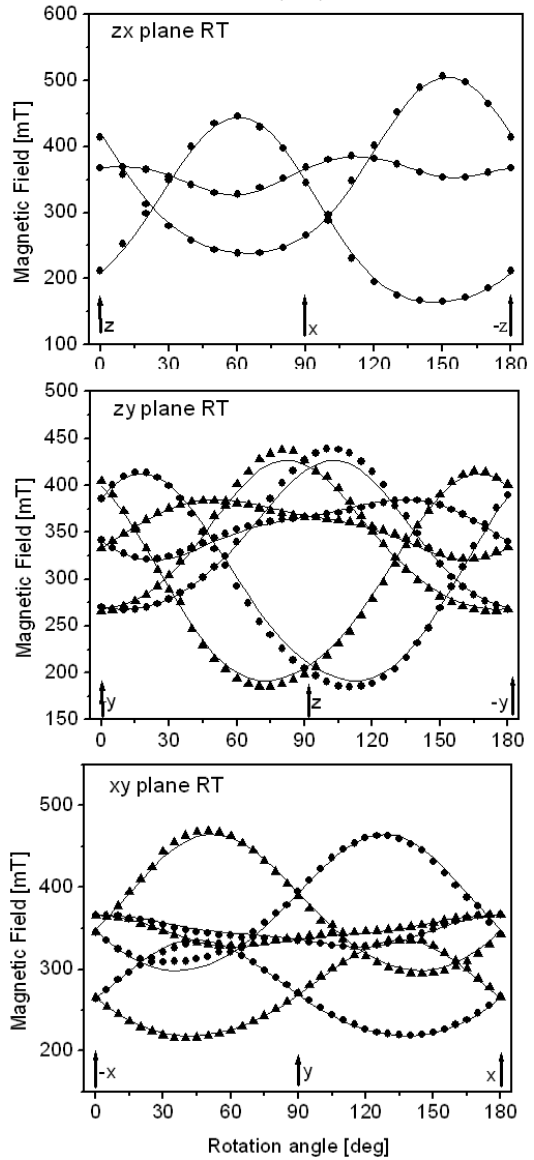

( b )
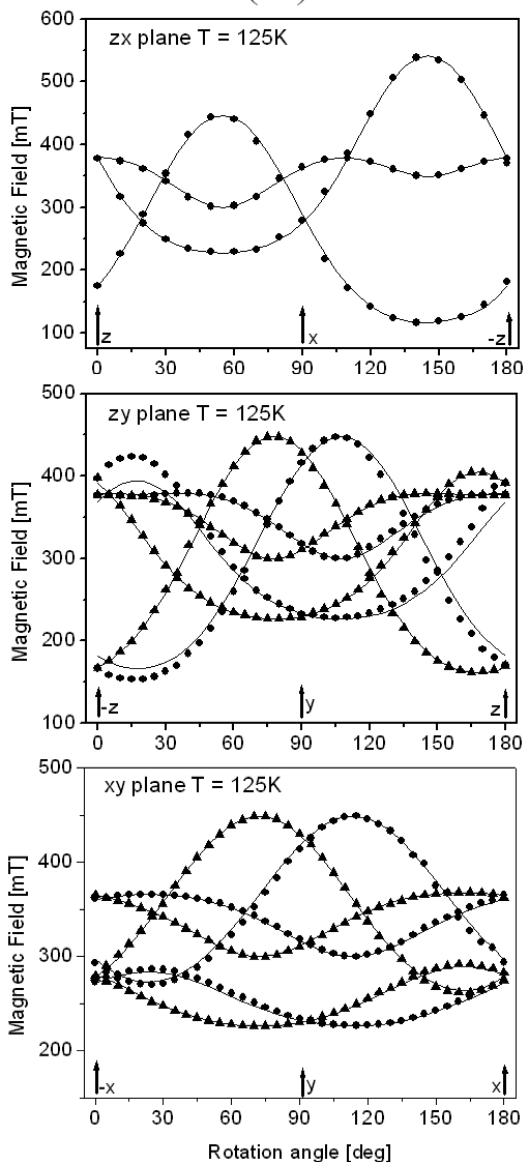

Fig. 1. The angular dependence of the $\mathrm{Cr}^{3+} \mathrm{EPR}$ spectra in the paraelectric phase in DMAGaS at X-band: (a) in ferroelastic phase at RT and (b) in ferroelectric phase at $125 \mathrm{~K}$. The experimental data of two different sets of the fine structure lines are represented by closed circles and triangles. The solid lines show the fit result with the parameters given in Table I.

and $z$-axes, respectively. The rotation patterns taken in the $x y, z y$, and $z x$ planes for both phases are shown in Fig. 1. The rotation pattern of the spectra show in the paraelectric and the ferroelectric phase two magnetically equivalent sets of lines in accordance with two chemically equivalent Ga sites in unit cell, which can be substituted by $\mathrm{Cr}^{3+}$ ions. For the each set, three allowed $\left(\Delta M_{s}= \pm 1\right)$ and three quasi-forbidden transitions $\left(\Delta M_{s}= \pm 2\right.$ and \pm 3$)$ between the four spin states: $3 / 2,1 / 2,-1 / 2$, and $-3 / 2$ due to the effective spin $S=3 / 2$ were clearly identified in the each plane. The quasi-forbidden transitions are not shown in Fig. 1. The spectra can be described by means of the spin Hamiltonian (SH): 


$$
\hat{H}=\beta \boldsymbol{B} \cdot \boldsymbol{g} \cdot \boldsymbol{S}+\frac{1}{3}\left(b_{2}^{0} O_{2}^{0}+b_{2}^{2} O_{2}^{2}\right),
$$

where $\boldsymbol{S}$ is the electron spin operator of the $\mathrm{Cr}^{3+}$ ion with the effective spin $S=3 / 2, \beta$ is the Bohr magneton, $\boldsymbol{B}$ is the external magnetic field, $\boldsymbol{g}$ is the spectroscopic splitting tensor and $b_{n}^{m} O_{n}^{m}$ terms represent crystal field contributions. The SH parameters were calculated with exact diagonalization of the energy matrices using a computer program provided by Grachev [16]. A procedure used for determination of SH parameters was the same as this one described in our former paper [15].

TABLE I

Spin-Hamiltonian parameters for $\mathrm{Cr}^{3+}$ in paraelectric (RT) and ferroelectric $(125 \mathrm{~K})$ phases of DMAGaS. Zero field splitting parameters are in $10^{-4} \mathrm{~cm}^{-1}$.

\begin{tabular}{c|c|c|c|c}
\hline \hline Temperature & $g$ & $b_{2}^{0}$ & $b_{2}^{2}$ & Reference \\
\hline RT & $1.982 \pm 0.002$ & $890 \pm 15$ & $386 \pm 15$ & present study \\
$125 \mathrm{~K}$ & $1.982 \pm 0.002$ & $1081 \pm 10$ & $20 \pm 10$ & present study \\
$124 \mathrm{~K}$ & - & 1068 & 38 & {$[14]$}
\end{tabular}

The SH parameters determined for both phases are listed in Table I together with previously reported values [14]. There is a very good agreement between the experimental line positions and the theoretical ones calculated by the use of optimized SH parameters (Fig. 1). Our experiments showed that no difference occurred between the fine structure parameters of two sites within the experimental errors. The structural data indicate that each $\mathrm{Cr}^{3+}$ ion is surrounded by six water molecules arranged in slightly disordered octahedral array. The effective local symmetry of crystal field at the site of $\mathrm{Cr}^{3+}$ ions can be characterized by the ratio $k=b_{2}^{2} / b_{2}^{0}$, which may be varied between 0 and 1 [17]. In particular, the parameter $b_{2}^{0}$ is the measure of the axially symmetric part of the zero-field splitting and $b_{2}^{2}$ is the measure of the part with orthorhombic symmetry. Thus, an increase in $k$ represents a deviation from axial symmetry to orthorhombic one. The estimated values of $k$, in system studied, vary from 0.434 at $300 \mathrm{~K}$ to 0.019 at $125 \mathrm{~K}$, so their change is much larger than observed in DMAAS [15]. The changes are seen both in the SH parameters themselves and also in the positions of the principal axes of the zero field splitting tensor if we compare their values in the both phases. The direction cosines of these tensors are given in Table II and III.

Temperature dependence of EPR spectra were studied from RT down to 110 K. Figure 2 shows thermal evolution of the $\mathrm{Cr}^{3+}$ spectra in DMAAS at selected orientation of the static magnetic field. Similarly as in DMAAS a strong broadening and then a line tripling is observed already in ferroelastic phase far above $T_{\mathrm{c} 1}=134 \mathrm{~K}$. The satellites of these triplets disappear in ferroelectric phase and lines narrow drastically on lowering the temperature (Fig. 3). Völkel et al. [10] 
TABLE II

Direction cosines of I and II site of $\mathrm{Cr}^{3+}$ ions in DMAGaS at $300 \mathrm{~K}$.

\begin{tabular}{c|c|c|c|c}
\hline \hline Site & \multirow{2}{*}{$\begin{array}{c}\text { Principal axes } \\
\text { of the complex }\end{array}$} & \multicolumn{3}{|c}{ Direction cosines } \\
\cline { 3 - 5 } & $X_{\mathrm{I}}$ & -0.257 & 0.764 & 0.591 \\
$\mathrm{I}$ & $Y_{\mathrm{I}}$ & 0.487 & -0.426 & 0.762 \\
& $Z_{\mathrm{I}}$ & 0.834 & 0.484 & -0.263 \\
\hline \multirow{3}{*}{$\mathrm{II}$} & $X_{\mathrm{II}}$ & -0.253 & 0.761 & -0.598 \\
& $Y_{\mathrm{II}}$ & -0.512 & 0.419 & 0.750 \\
& $Z_{\mathrm{II}}$ & 0.821 & 0.496 & 0.283
\end{tabular}

TABLE III

Direction cosines of I and II site of $\mathrm{Cr}^{3+}$ ions in DMAGaS at $125 \mathrm{~K}$.

\begin{tabular}{c|c|c|c|c}
\hline \hline Site & \multirow{2}{*}{$\begin{array}{c}\text { Principal axes } \\
\text { of the complex }\end{array}$} & \multicolumn{3}{|c}{ Direction cosines } \\
\cline { 3 - 5 } & $X_{\mathrm{I}}$ & -0.227 & 0.609 & 0.759 \\
\hline \multirow{3}{*}{$\mathrm{I}$} & $Y_{\mathrm{I}}$ & -0.552 & 0.562 & -0.616 \\
& $Z_{\mathrm{I}}$ & 0.802 & 0.559 & -0.209 \\
\hline \multirow{3}{*}{$\mathrm{II}$} & $X_{\mathrm{II}}$ & 0.031 & -0.394 & 0.918 \\
& $Y_{\text {II }}$ & -0.604 & 0.724 & 0.332 \\
& $Z_{\text {II }}$ & 0.796 & 0.566 & 0.216
\end{tabular}

gives simple explanation of the observed line tripling taking into account influence of four possible configurations of two neighbouring DMA groups on water octahedron with the chromium probes substitution at the gallium sites. Thus, the amino group has four equilibrium positions, which are realized in two groups with different probability. This behaviour of the spectra is in good agreement with the four states model proposed for description of the phase transition crystals of DMAMeS $(\mathrm{Me}=\mathrm{Al}, \mathrm{Ga})$ type by Stasyuk and Velychko [18]. The temperature dependence of the line width corresponding to the $(-1 / 2 \leftrightarrow-3 / 2)$ transition in DMAGaS with magnetic field making an angle $(\boldsymbol{B}, \boldsymbol{z})=30^{\circ}$ is shown in Fig. 3. These line width changes confirm the order-disorder character of the ferroelectric phase transition. At $T>T_{\mathrm{c} 1}$ DMA groups are dynamically disordered, executing random flips around the axis connecting the two methyl oxygens. Below $T_{\mathrm{c} 1}$ the DMA molecule freezes in one position and the spontaneous dielectric polarization $P_{\mathrm{S}}$ occurs in the $m$-plane. Thus, the DMA group represents the basic reversible electric dipole and its ordering creates $P_{\mathrm{S}}$ below $T_{\mathrm{c} 1}$. Figure 4 shows the temperature dependence of the spectra in selected orientation in the whole temperature 


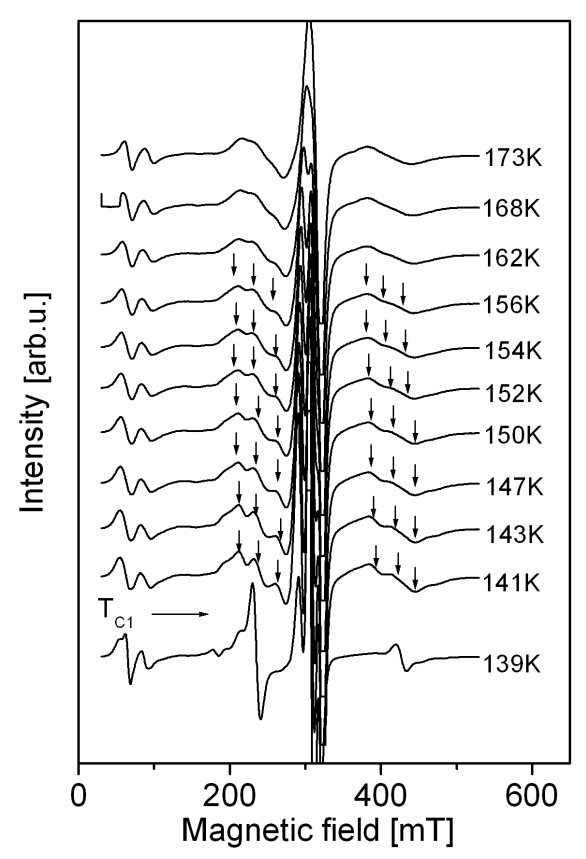

Fig. 2. Temperature dependence of the $\mathrm{Cr}^{3+}$ spectrum in DMAGaS near the phase transition into the ferroelectric phase measured at X-band with the static magnetic field $B$ making an angle $(\boldsymbol{B}, \boldsymbol{z})=50^{\circ}$. Characteristic triplet lines are marked by arrows.

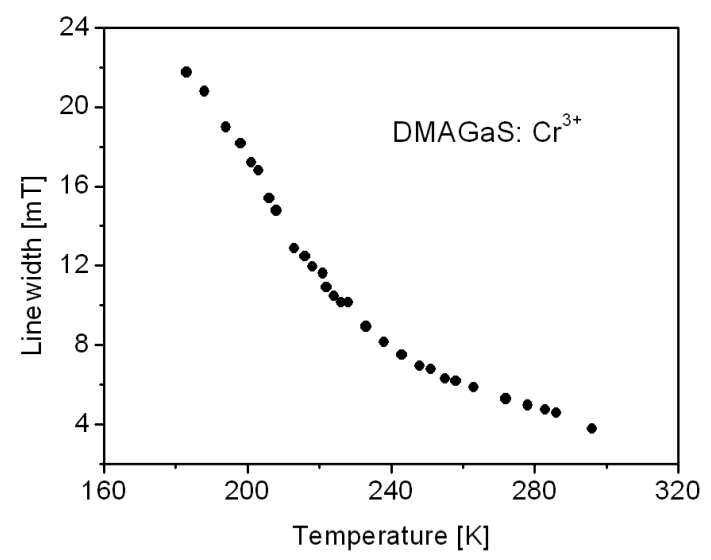

Fig. 3. Temperature dependence of the high field line width of $\mathrm{Cr}^{3+}(-1 / 2 \leftrightarrow-3 / 2)$ in DMAGaS with the orientation of the magnetic field applied as in Fig. 4.

range from $\mathrm{RT}$ down to $110 \mathrm{~K}$. At $T_{\mathrm{c} 2}=116 \mathrm{~K}$ the crystal undergoes another transition into the non-ferroelectric low temperature phase and the spectrum abruptly changes. It is associated with large shifts of line positions, striking line broadening and multiplication of the number of lines. Völkel et al. [14] have studied DMAGaS 


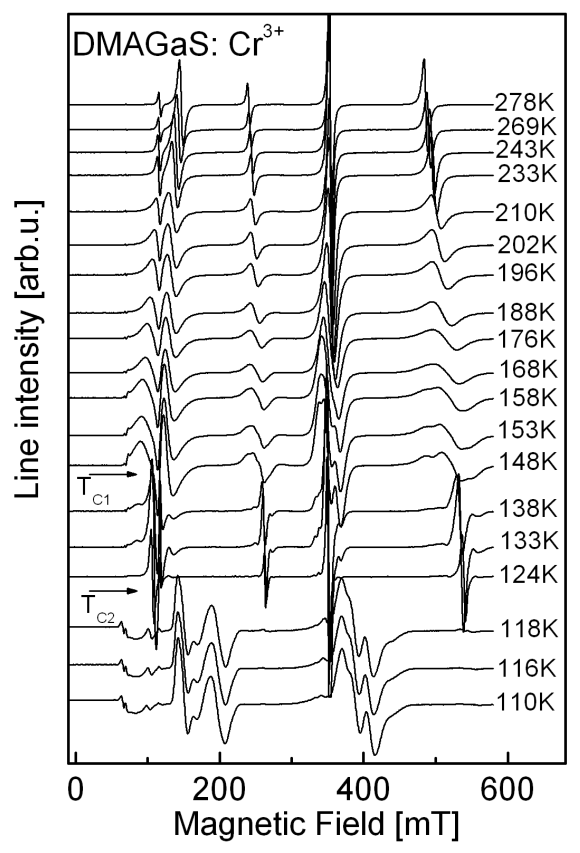

Fig. 4. Temperature dependence of the $\mathrm{Cr}^{3+}$ spectrum in DMAGaS measured at X-band with the static magnetic field $B$ making an angle $(\boldsymbol{B}, \boldsymbol{z})=30^{\circ}$. The phase transition temperatures $T_{\mathrm{c} 1}$ and $T_{\mathrm{c} 2}$ are shown.

below $T_{\mathrm{c} 2}=116 \mathrm{~K}$ and explained temperature behaviour of the spectra assuming existence of the modulated phase in the crystal with commensurate region and discommensurations before it becomes antiferroelectric at $T^{*}=60 \mathrm{~K}$. Our studies of the low temperature modulated and non-polar phases are in progress.

\section{References}

[1] L.F. Kirpichnikova, A. Pietraszko, K. Lukaszewicz, L.A. Shuvalov, V.V. Dolbinina, L.M. Yakovleva, Crystallogr. Rep. 39, 990 (1994).

[2] L.F. Kirpichnikova, L.A. Shuvalov, N.R. Ivanov, B.N. Prasolov, E.F. Andreyev, Ferroelectrics 96, 313 (1989).

[3] A. Pietraszko, K. Lukaszewicz, L.F. Kirpichnikova, Pol. J. Chem. 67, 1877 (1993).

[4] A. Pietraszko, K. Lukaszewicz, L.F. Kirpichnikova, Pol. J. Chem. 69, 922 (1995).

[5] V. Kapustianik, M. Bublyk, I. Polovinko, S. Sveleba, Z. Trybula, E. Andreyev, Phase Transit. 49, 231 (1994).

[6] V. Kapustianik, M. Fally, H. Kabelka, H. Warhanek, J. Phys., Condens. Matter 9, 723 (1997).

[7] S. Dacko, Z. Czapla, Ferroelectrics 189, 143 (1996).

[8] V.Yu. Kazimirov, V.A. Sarin, M.B. Smirnov, L.A. Shuvalov, Ferroelectrics 299, 59 (2004). 
[9] N. Alsabbagh, D. Michel, Z. Czapla, J. Furtak, Phys. Status Solidi A 167, 77 (1998).

[10] G. Völkel, N. Alsabbagh, R. Böttcher, D. Michel, B. Milsch, Z. Czapla, J. Furtak, J. Phys., Condens. Matter 12, 4553 (2000).

[11] G. Völkel, R. Böttcher, Z. Czapla, D. Michel, Ferroelectrics 268, 181 (2002).

[12] W. Bednarski, S. Waplak, L.F. Kirpichnikova, J. Phys., Condens. Matter 11, 1567 (1999).

[13] J. Dolinšek, M. Klanjšek, D. Arčon, Hae Jin Kim, J. Seliger, V. Žagar, Phys. Rev. B 59, 3640 (1999).

[14] G. Völkel, R. Böttcher, D. Michel, Z. Czapla, Phys. Rev. B 67, 24111 (2003).

[15] R. Hrabański, M. Janiec, M. Jackowska, V. Kapustianik, Appl. Magn. Reson. 27, 19 (2004).

[16] V.G. Grachev, Visual EPR program v. 2.08, Fachbereich Physik Universität Osnabrück, Osnabrück 1998.

[17] C. Rudowicz, J. Phys., Condens. Matter 12, L417 (2000).

[18] I.V. Stasyuk, O.V. Velychko, Phase Transit. 73, 483 (2001). 